\title{
SKA1 overexpression is associated with the prognosis of esophageal squamous cell carcinoma and regulates cell proliferation and migration
}

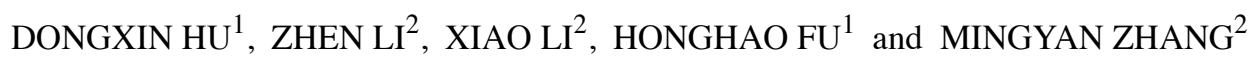 \\ Departments of ${ }^{1}$ Thoracic Surgery and ${ }^{2}$ Gastroenterology, Shandong Provincial Hospital \\ Affiliated to Shandong University, Jinan, Shandong 250021, P.R. China
}

Received June 11, 2019; Accepted August 26, 2019

DOI: $10.3892 /$ ijmm.2019.4343

\begin{abstract}
Spindle and kinetochore-associated protein 1 (SKA1), a microtubule-binding subcomplex of the outer kinetochore, is essential for complete chromosomal separation. SKA1 has been suggested as a potential biomarker for various types of cancer. However, the exact role of SKA1 in esophageal squamous cell carcinoma (ESCC) remains unclear. The present study investigated whether SKA1 affects the biological behavior of ESCC. The expression of SKA1 in ESCC tissues was measured using immunohistochemistry and reverse transcription-quantitative polymerase chain reaction. In addition, a SKA1-silencing lentivirus was constructed, which was transfected into TE-1 cells to establish stable SKA1-knockdown TE-1 cells. Proliferation was analyzed using a Celigo image cytometer and a MTS assay. Cell cycle progression and apoptosis were analyzed by flow cytometry, while cell migration was assessed using a Transwell assay. SKA1 was significantly overexpressed in ESCC tissues, and SKA1 overexpression was significantly associated with differentiation, pathological $\mathrm{N}$ stage and pathological tumor-node-metastasis stage. SKA1 was determined to be an independent prognostic factor for ESCC. Furthermore, SKA1 was significantly overexpressed in ESCC cells, and SKA1-silencing inhibited cell proliferation and migration, arrested the cell cycle and promoted cell apoptosis. In summary, SKA1 may serve as a potential therapeutic target and prognostic biomarker for ESCC.
\end{abstract}

Correspondence to: Dr Mingyan Zhang, Department of Gastroenterology, Shandong Provincial Hospital Affiliated to Shandong University, 324 Jing 5 Road, Jinan, Shandong 250021, P.R. China

E-mail: 312617scott@163.com

Abbreviations: SKA1, spindle and kinetochore-associated protein 1; ESCC, esophageal squamous cell carcinoma; TNM, tumor-node-metastasis; EC, esophageal cancer

Key words: spindle and kinetochore-associated protein 1, prognosis, esophageal squamous cell carcinoma, proliferation, migration

\section{Introduction}

Esophageal cancer (EC) is one of the most common types of malignant tumor and was the sixth most common cause of cancer-associated mortality worldwide in 2018 (1). EC has the highest prevalence rate and was the fourth most common cause of cancer-associated mortality in China in $2015(2,3)$. Esophageal squamous cell carcinoma (ESCC) is the main histological type of EC, accounting for $88 \%$ of EC cases worldwide (4). Although a combination of surgery, radiotherapy and chemotherapy has demonstrated good results for ESCC treatment, the prognosis of patients remains unsatisfactory and the 5-year survival rate was $20.9 \%$ in China in 2015 (5). The etiology of EC includes tobacco smoking, alcohol drinking, low intake of fruits and vegetables, gastroesophageal reflux and an involvement of susceptibility-related genes $(6,7)$. Despite advancements in the search for diagnostic and prognostic markers, no specific biomarker has regularly been used for ESCC diagnosis $(8,9)$. Therefore, it is imperative to identify reliable therapeutic and prognostic molecular targets for patients with ESCC as a guide for further adjuvant treatment.

Spindle and kinetochore-associated protein 1 (SKA1) belongs to the family of spindle- and centromere-binding proteins and is a microtubule-binding protein (10). The complex is essential for stabilizing the kinetochore-spindle microtubule attachment during mitosis (11). The absence of SKA1 protein may result in severe chromosomal separation defects (11). Previously, numerous studies have highlighted the association between high SKA1 expression and poor prognosis in cancer $(12,13)$. However, the prognostic value and functions of SKA1 in ESCC remain unclear. The present study aimed to verify the expression of SKA1 in 110 patients with ESCC through immunohistochemistry (IHC), and investigate the role and functions of SKA1 in ESCC.

\section{Materials and methods}

Patients and tissues. A total of 110 patients with complete clinical records who were treated at the Department of Thoracic Surgery, Provincial Hospital Affiliated to Shandong University (Jinan, China) between January 2011 and January 2013 were enrolled for this retrospective study. The patients included 
89 males and 21 females, with a mean age of 61 years (range, 31-79 years). The clinical information of all patients is presented in Table I. The inclusion criteria were as follows: i) Patients subjected to Ivor-Lewis esophagogastrectomy with thoracic and abdominal two-field lymph node dissection (14) to achieve complete tumor resection as per the 2009 Union for International Cancer Control (UICC) standard for midthoracic ESCC; ii) patients restaged according to the 2009 UICC tumor-node-metastasis (TNM) staging guidelines for EC; iii) no residual cancer cells under the upper and lower cutting edge, and a lateral margin without residual focus, as demonstrated by pathological examination; iv) >12 lymph node dissections; v) no preoperative chemotherapy or radiotherapy; vi) no severe preoperative complications; and vii) at least a complete 3 -year follow-up review and first specific recurrence location recorded. ESCC tissue and corresponding healthy esophageal mucosa (CHEM) tissue samples were collected from each patient. The CHEM tissue samples were obtained $5 \mathrm{~cm}$ from the edge of the ESCC tissues, and they were examined by light microscopy to confirm the absence of necrosis, deterioration or tumor.

IHC analysis. IHC analysis was conducted as described previously (15). Briefly, ESCC and CHEM tissues were fixed with $4 \%$ paraformaldehyde at $4^{\circ} \mathrm{C}$ for $24 \mathrm{~h}$, paraffin-embedded and cut into $5-\mu \mathrm{m}$ sections. Following blocking with goat serum for $30 \mathrm{~min}$ at $25^{\circ} \mathrm{C}$ for $40 \mathrm{~min}$, sections were incubated with anti-SKA1 primary antibody (1:50; cat. no. ab91550; Abcam) at $37^{\circ} \mathrm{C}$ for $1 \mathrm{~h}$. After three washes with PBS, the sections incubated with a biotin goat anti-rabbit IgG H\&L secondary antibody (1:2,000; cat. no. ab6720; Abcam) for $30 \mathrm{~min}$ at $37^{\circ} \mathrm{C}$, followed by incubation with streptavidin-horseradish peroxidase for $5 \mathrm{~min}$ at $37^{\circ} \mathrm{C}$. Subsequently, 3,3'-diaminobenzidine was added for visualization after washing with PBS three times. Finally, the sections were counterstained with hematoxylin at $25^{\circ} \mathrm{C}$ for $1 \mathrm{~min}$, air-dried and mounted on a cover slip. SKA1 expression was observed under a light microscope at three magnifications (x100, x200 and x400).

The immunohistochemical score (IHS) consisted of the proportion score (percentage of positively stained cells) and the staining intensity score. The proportion score was described as follows: $0(<5 \%), 1(5-24 \%), 2(25-49 \%), 3(50-74 \%)$ and $4(75-100 \%)$. The staining intensity score was calculated as follows: 0 (negative), 1 (weak), 2 (moderate) and 3 (strong). The proportion score was multiplied by the staining intensity score to obtain an IHS for each section. SKA1 expression was rated as follows: High expression, HIS $\geq 4$; and low expression, HIS <4. Two experienced pathologists blinded to the patient data independently scored the samples and agreed on a result through reanalysis and discussion.

Follow-up after surgery. All patients were regularly followed-up every 3-6 months after surgery for a general physical examination, abdominal ultrasound, chest and upper abdominal contrast-enhanced computed tomography scan, and positron emission tomography. Biopsies were used based on specific imaging and clinical examinations. The follow-up was completed in January 2017 with a maximum follow-up period of 60 months.

Cell culture. The human ESCC cell lines TE-1, EC9706 and Eca-109, a human esophageal epithelial cell line HET-1A and
293T cells were purchased from the Cell Bank of Chinese Academy of Sciences. ESCC cell lines and HET-1A were cultured in RPMI-1640 medium (Gibco; Thermo Fisher Scientific, Inc.), and 293T cells were cultured in Dulbecco's modified Eagle's medium (Gibco; Thermo Fisher Scientific, Inc.), which were both supplemented with $10 \%$ fetal bovine serum (Gibco; Thermo Fisher Scientific, Inc.), $100 \mathrm{U} / \mathrm{ml}$ penicillin $\mathrm{G}$ and $100 \mu \mathrm{g} / \mathrm{ml}$ streptomycin (Sigma-Aldrich; Merck $\mathrm{KGaA}$ ). All cell lines were incubated at $37^{\circ} \mathrm{C}$ in $5 \% \mathrm{CO}_{2}$.

Construction of recombinant lentivirus and infection. A short hairpin RNA (shRNA) targeting human SKA1 (SKA1-shRNA; 5'-GGAGATGAGATCATTGTAA-3') was inserted into the lentivirus expression plasmid pFH-L (Genechem, Inc.). Non-silencing shRNA (5'-GGAGATGAGATCATTGTAA-3') was used as a control (control-shRNA). Recombinant pFH-L plasmids $(20 \mu \mathrm{g})$ and two other helper plasmids [pVSVG-I $(15 \mu \mathrm{g})$ and pCMV-R8.92 $(10 \mu \mathrm{g})]$ (Shanghai Hollybio) were transfected into $293 \mathrm{~T}$ cells using Lipofectamine ${ }^{\circledR} 2000$ (Invitrogen; Thermo Fisher Scientific, Inc.) to package lentivirus expressing SKA1-shRNA or control-shRNA. After $48 \mathrm{~h}$, the supernatant was collected and the concentrated supernatant was used to infect TE-1 cells (multiplicity of infection, 10) following titer determination. TE-1 cells infected with lentivirus expressing SKA1-shRNA or control-shRNA were termed shSKA1 or shCtrl, respectively.

$R N A$ extraction and reverse transcription-quantitative polymerase chain reaction ( $R T-q P C R$ ). TE-1 cells of the shSKA1 or shCtrl groups were cultured for $48 \mathrm{~h}$ and cells were then collected for RNA extraction. TRIzol ${ }^{\circledR}$ (Invitrogen; Thermo Fisher Scientific, Inc.) was used according to the manufacturer's protocol to extract total RNA from tumor tissues and cultured cells. Complementary DNA (cDNA) was prepared using superscript III reverse transcriptase (Invitrogen; Thermo Fisher Scientific, Inc.), according to the manufacturer's protocol. qPCR was performed using SYBR Green qPCR SuperMix (Invitrogen; Thermo Fisher Scientific, Inc.) to detect mRNA expression with the following conditions: $95^{\circ} \mathrm{C}$ for $2 \mathrm{~min}$; 40 cycles of $95^{\circ} \mathrm{C}$ for $15 \mathrm{sec}$ and $60^{\circ} \mathrm{C}$ for $1 \mathrm{~min}$. GAPDH cDNA was amplified and used as an internal standard. The primers used were as follows: SKA1 forward, 5'-TTCCCATTTGCCTCAAGTAACAG-3' and reverse, 5'-GGAACACCATTGAACTCATCACAAG-3'; and GAPDH forward, 5'-GCACCGTCAAGGCTGAGAAC-3' and reverse, 5'-TGGTGAAGACGCCAGTGGA-3'. Relative expression was calculated using the $2^{-\Delta \Delta \mathrm{Cq}}$ method (16).

Western blot analysis. TE-1 cells of the shSKA1 or shCtrl groups were cultured for $48 \mathrm{~h}$ and cells were then collected for total protein extraction. Total protein was extracted using ice-cold radioimmunoprecipitation assay extraction and lysis buffer (Takara Bio, Inc.) containing a proteinase inhibitor cocktail (Takara Bio, Inc.). The concentration of total protein was determined using a BCA Protein assay kit (Nanjing KeyGen Biotech Co., Ltd.), according to the manufacturer's protocol. The extracted proteins (30 $\mu \mathrm{g})$ were separated by $10 \%$ SDS-PAGE and the separated protein bands were transferred onto nitrocellulose membranes. The membranes were incubated with rabbit anti-SKA1 antibody (1:500; cat. no. ab91550; Abcam) and rabbit anti-GAPDH antibody (1:10,000; cat. no. ab181602; Abcam) 
Table I. Association of SKA1 expression with clinicopathological features of patients with ESCC.

\begin{tabular}{|c|c|c|c|c|}
\hline \multirow[b]{2}{*}{ Characteristic } & \multirow[b]{2}{*}{ Total $(\mathrm{n}=110)$} & \multicolumn{2}{|c|}{ SKA1 expression level } & \multirow[b]{2}{*}{ P-value } \\
\hline & & Low $(n=42)$ & High $(n=68)$ & \\
\hline Age, years & & & & 0.152 \\
\hline$<50$ & 23 & 12 & 11 & \\
\hline$\geq 50$ & 87 & 30 & 57 & \\
\hline Sex & & & & 0.322 \\
\hline Male & 89 & 32 & 57 & \\
\hline Female & 21 & 10 & 11 & \\
\hline Tumor size, mm & & & & 0.576 \\
\hline$<30$ & 20 & 8 & 12 & \\
\hline $30-50$ & 52 & 22 & 30 & \\
\hline$>50$ & 38 & 12 & 26 & \\
\hline Differentiation degree & & & & $0.018^{\mathrm{a}}$ \\
\hline High & 16 & 11 & 5 & \\
\hline Moderate & 52 & 19 & 33 & \\
\hline Low & 42 & 12 & 30 & \\
\hline Pathological T stage & & & & 0.527 \\
\hline $\mathrm{T} 1$ & 4 & 2 & 2 & \\
\hline $\mathrm{T} 2$ & 26 & 12 & 14 & \\
\hline $\mathrm{T} 3+\mathrm{T} 4$ & 80 & 28 & 52 & \\
\hline Pathological N stage & & & & $0.017^{\mathrm{a}}$ \\
\hline N0 & 28 & 16 & 12 & \\
\hline $\mathrm{N} 1+\mathrm{N} 2$ & 82 & 26 & 56 & \\
\hline Pathological TNM stage & & & & $0.045^{\mathrm{a}}$ \\
\hline $\mathrm{I}+\mathrm{II}$ & 30 & 16 & 14 & \\
\hline III+IV & 80 & 26 & 54 & \\
\hline
\end{tabular}

${ }^{\mathrm{a}} \mathrm{P}<0.05$ as determined by a $\chi^{2}$ test. TNM, tumor-node-metastasis

at $4^{\circ} \mathrm{C}$ overnight, followed by treatment with horseradish peroxidase-conjugated goat anti-rabbit IgG $\mathrm{H} \& \mathrm{~L}$ secondary antibody $\left(1: 10,000\right.$; SouthernBiotech) for $40 \mathrm{~min}$ at $25^{\circ} \mathrm{C}$. The protein bands were visualized using enhanced chemiluminescence substrate (Thermo Scientific Inc.). The expression of all proteins was normalized against that of GAPDH.

Cell count assay. TE-1 cells of the shSKA1 and shCtrl groups were seeded into 96-well plates (1,500 cells/well) and incubated at $37^{\circ} \mathrm{C}$ under $5 \% \mathrm{CO}_{2}$ for 4 days. The cell count assay was performed using a Celigo Imaging Cytometer (Nexcelom Bioscience), according to the manufacturer's protocol. The captured cell images were analyzed using Celigo software (Nexcelom Bioscience).

Cell viability assay. CellTiter $96^{\circledR}$ AQueous One Solution assay kit was used to detect cell viability. Briefly, TE-1 cells ( $2 \times 10^{3} /$ well) of the shSKA1 and shCtrl groups were seeded in 96-well plates. After 1, 2 or 3 days of incubation, the cells were treated with $5 \mathrm{mg} / \mathrm{ml}$ MTS reagent and incubated for $4 \mathrm{~h}$ at $37^{\circ} \mathrm{C}$. Following incubation, the supernatant was used to measure the optical density (OD) at a wavelength of $490 \mathrm{~nm}$ using a microplate reader. All experiments were performed in triplicate. Cell viability was calculated using the following equation: Cell viability $(\%)=(\mathrm{OD}$ value of tested group/OD value of shCtrl group) $\mathrm{x} 100 \%$.

Cell cycle analysis. TE-1 cells of the shSKA1 and shCtrl groups were seeded in six-well plates ( $4 \times 10^{5} /$ well). After 2 days, the cell cycle was analyzed using propidium iodide (Sigma-Aldrich; Merck KGaA) staining for $30 \mathrm{~min}$ at $37^{\circ} \mathrm{C}$ and flow cytometry (Cytomics FC 500 MPL, Beckman Coulter, Inc.). Results were analyzed using ModfitLT, version 2.0 (Verity Software House Inc.). All experiments were performed in triplicate.

Apoptotic assay. The ApoScreen Annexin V Apoptosis kit (Southern Biotech) was used to label apoptotic cells, according to the manufacturer's protocol. Following culture for $48 \mathrm{~h}$, TE-1 cells of the shSKA1 or shCtrl groups were harvested and stained with Annexin V-APC, followed by flow cytometry analysis (FACSCalibur; Becton, Dickinson and Company) after $15 \mathrm{~min}$ of incubation at $25^{\circ} \mathrm{C}$. Results were analyzed using BD FACSDiva software (version 8.0; Becton, Dickinson and Company). All experiments were performed in triplicate. 
A

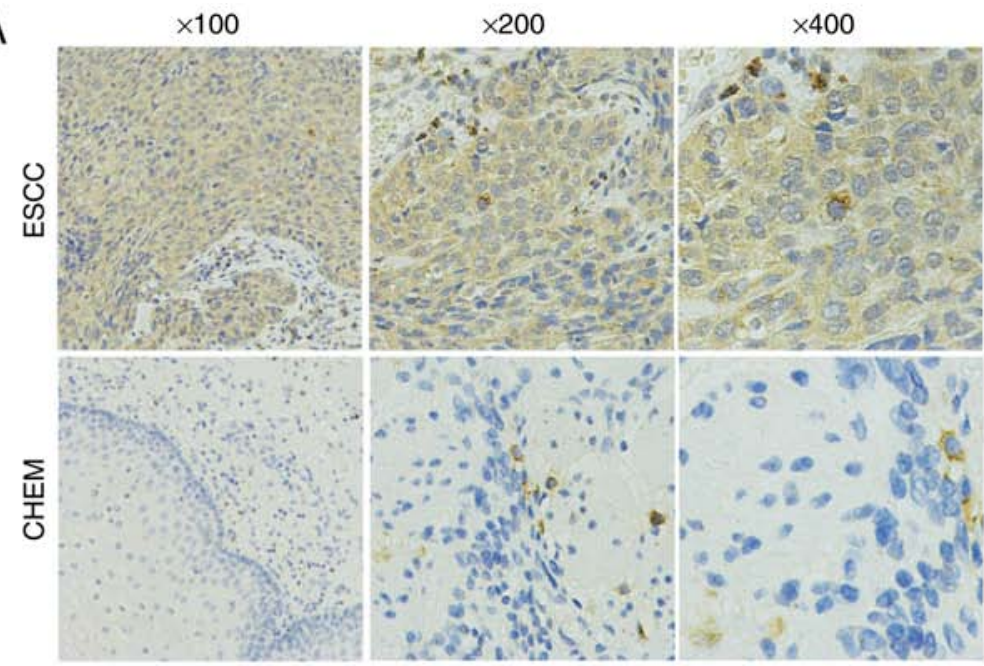

B

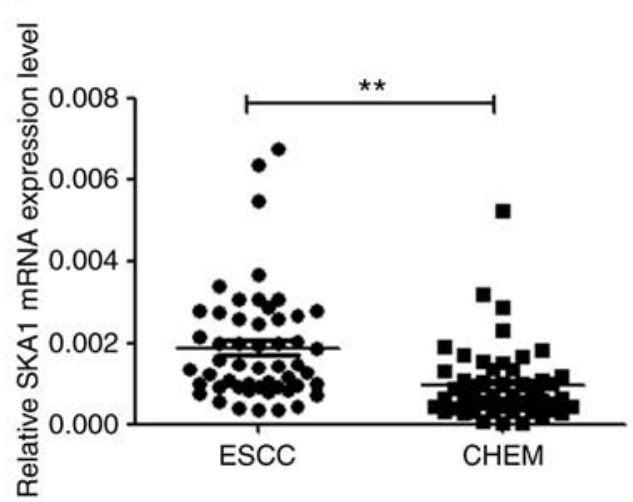

Figure 1. SKA1 is significantly overexpressed in ESCC tissues. (A) SKA1 expression was detected by immunohistochemistry staining of ESCC tissues $(n=110)$ and the CHEM tissues $(n=110)$. (B) SKA1 mRNA expression levels in ESCC tissues $(n=110)$ and CHEM ( $=110)$ tissues were measured by reverse transcription-quantitative polymerase chain reaction. ${ }^{* *} \mathrm{P}<0.01$. CHEM, corresponding healthy esophageal mucosa; ESCC, esophageal squamous cell carcinoma; SKA1, spindle and kinetochore-associated protein 1.

Migration assay. TE- 1 cells $\left(4 \times 10^{4}\right)$ of the shSKA1 and shCtrl groups were seeded onto the upper chamber of a Transwell plate in serum-free RPMI-1640 medium. RPMI-1640 medium (600 $\mu \mathrm{l})$ with $20 \%$ fetal bovine serum (Gibco; Thermo Fisher Scientific, Inc.) was added to the lower chamber of the Transwell plate. After $48 \mathrm{~h}$, the migration of cells to the lower chamber of the Transwell plate was determined. Cells were stained with $0.1 \%$ crystal violet in $20 \%$ ethanol for $10 \mathrm{~min}$ at $25^{\circ} \mathrm{C}$, and the number of cells was counted in five independent fields under a light microscope (magnification, $x 200$ ). All experiments were performed in triplicate.

Statistical analysis. SPSS 19.0 software (SPSS, Inc.) was used for all statistical analyses. The significant difference in SKA1 expression between tumor and para-tumor samples was evaluated using a $\chi^{2}$ test. The Kaplan-Meier method was used to analyze the survival rate and the significance of the survival difference was calculated using a log-rank test. Independent prognostic factors were determined through Cox regression multivariate analysis. Statistical comparisons between two groups were analyzed using an unpaired Student's t-test. Statistical analysis for more than two groups was performed using one-way ANOVA followed by a post-hoc least significant difference test. $\mathrm{P}<0.05$ was considered to indicate a statistically significant difference.

\section{Results}

SKAl is significantly overexpressed in ESCC tissues. As presented in Fig. 1A, SKA1 expression was predominantly detected in the cytoplasm of tumor cells. SKA1 protein expression was markedly higher in ESCC tissues compared with in CHEM tissues (Fig. 1A). Of the 110 samples, 68 (61.8\%) tumor samples and only 29 (26.4\%) CHEM samples exhibited a high SKA1 expression. In addition, SKA1 mRNA expression was significantly higher in ESCC tissues compared with in CHEM tissues (Fig. 1B).
SKA1 overexpression is associated with clinicopathological parameters. The association between SKA1 expression and clinicopathological parameters is presented in Table I. No significant associations were identified between SKA1 expression and age, sex, tumor size or pathological T stage. However, SKA1 overexpression was significantly associated with differentiation, pathological $\mathrm{N}$ stage and pathological TNM stage (Table I).

SKA1 protein overexpression is associated with the prognosis of patients with ESCC. Follow-up data were available for all patients included in the present study. The results of Kaplan-Meier analysis indicated that the overall 5-year survival rate was $27.27 \%$. Patients with high SKA1 protein expression had a 5-year survival rate of $17.64 \%$, compared with a survival rate of $42.85 \%$ for patients with low SKA1 expression $(\mathrm{P}<0.01$; Fig. 2). The results of multivariate analysis revealed that pathological TNM stage and SKA1 protein overexpression were independent prognostic factors for ESCC (Table II).

SKAl is significantly overexpressed in ESCC cells. The mRNA expression of SKA1 in the human ESCC cell lines TE-1, EC9706 and Eca-109, and a human esophageal epithelial cell line HET-1A was investigated through RT-qPCR analysis. It was identified that the expression of SKA1 mRNA was significantly higher in TE-1, EC9706 and Eca-109 cells compared with in HET-1A cells (Fig. 3). Among the ESCC cell lines, the SKA1 mRNA level was highest in TE-1 cells. Therefore, TE- 1 cells were selected for the following assays.

Expression of SKA1 is downregulated in TE-1 cells following transfection with lentivirus-mediated shRNA. To evaluate the functions of SKA1 in ESCC cells, SKA1 expression was knocked-down by lentivirus-mediated shRNA transfection. The mRNA and protein expression levels of SKA1 were evaluated using RT-qPCR and western blot analysis, respectively. In comparison with the shCtrl group, the cells transfected with shSKA1 demonstrated a marked reduction in the mRNA 
Table II. Results of multivariate Cox regression analysis.

\begin{tabular}{lcccccc}
\hline Variable & $\mathrm{B}$ & $\mathrm{SE}$ & Wald & P-value & HR & 95\% CI \\
\hline Differentiation & 0.103 & 0.185 & 0.310 & 0.578 & 1.109 & $0.771-1.594$ \\
Pathological N stage & 0.484 & 0.349 & 1.921 & 0.166 & 1.622 & $0.819-3.215$ \\
Pathological TNM stage & 0.823 & 0.323 & 6.476 & 0.011 & 2.278 & $1.208-4.293$ \\
SKA1 expression & 0.553 & 0.255 & 4.724 & 0.030 & 1.739 & $1.056-2.864$
\end{tabular}

SE, standard error; HR, hazard ratio; CI, confidence interval; TNM, tumor-node-metastasis; SKA1, spindle and kinetochore-associated protein 1.

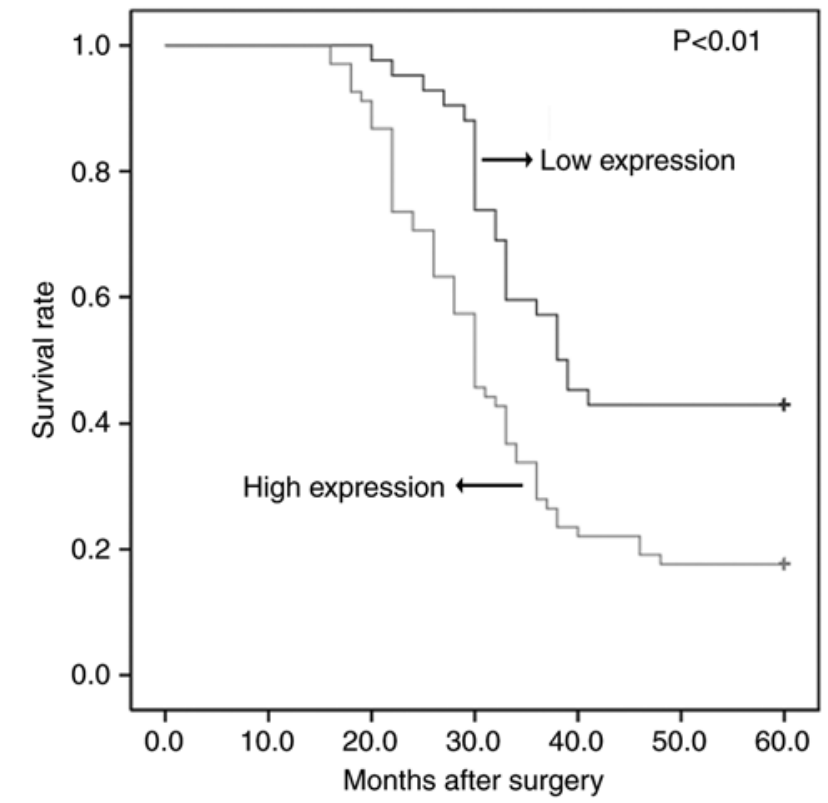

Figure 2. Kaplan-Meier survival analysis. Patients with ESCC from the high SKA1 expression group exhibited significantly lower survival rates compared with those from the low SKA1 expression group. ESCC, esophageal squamous cell carcinoma; SKA1, spindle and kinetochore-associated protein 1.

and protein expression levels of SKA1 (Fig. 4). Therefore, lentivirus-mediated shRNA knockdown was demonstrated to serve as an effective strategy to knockdown SKA1 expression in EC cells.

SKA1-knockdown suppresses the proliferation of TE-1 cells. Celigo image cytometry analysis and an MTS assay were used to evaluate the effect of SKA1-knockdown on the proliferation ability of TE-1 cells. As presented in Fig. 5A and B, the cell number in the shSKA1 group was significantly lower compared with that in the shCtrl group at days 1-4. In addition, the results of the MTS assays demonstrated that the percentage of cell viability in the shSKA1 group was significantly lower compared with that of the shCtrl group at days 1,2 and 3 (Fig. 5).

SKA1-knockdown arrests cell cycle progression and induces the apoptosis of TE-1 cells. It was identified that the proportion of cells in the G1 and S phases was significantly higher in the shSKA1 group compared with in the shCtrl group (Fig. 6A). In addition, apoptosis analysis revealed that the apoptosis rate

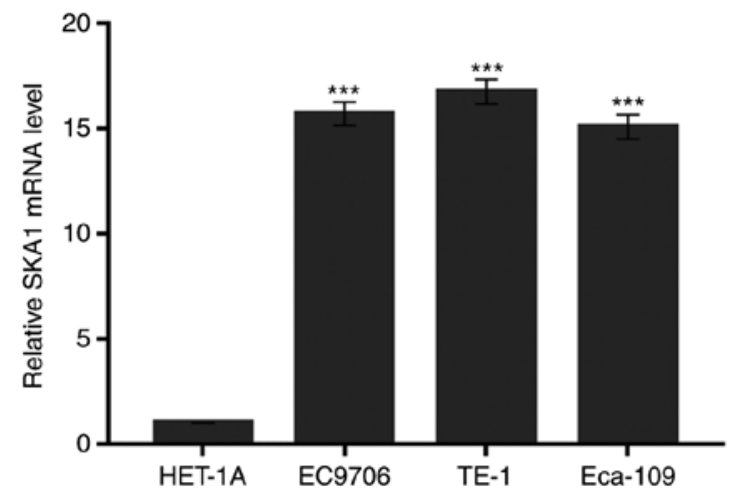

Figure 3. SKA1 is significantly overexpressed in ESCC cells. The mRNA expression levels of SKA1 in the human ESCC cell lines TE-1, EC9706 and Eca-109, and a human esophageal epithelial cell line HET-1A were measured by reverse transcription-quantitative polymerase chain reaction. ${ }^{* * *} \mathrm{P}<0.001$ vs. HET-1A cells. ESCC, esophageal squamous cell carcinoma; SKA1, spindle and kinetochore-associated protein 1.

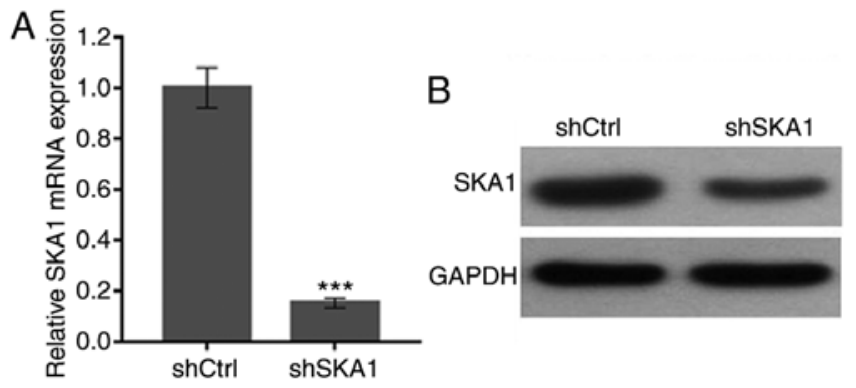

Figure 4. Expression of SKA1 in TE-1 cells is downregulated following lentivirus-mediated shRNA transfection. SKA1 (A) mRNA and (B) protein expression levels were analyzed using RT-qPCR and western blotting, respectively. ${ }^{* * *} \mathrm{P}<0.001$ vs. shCtrl. SKA1, spindle and kinetochore-associated protein 1; shRNA, short hairpin RNA; shCtrl, cells transfected with control shRNA; shSKA1, cells transfected with SKA1 shRNA.

was significantly higher in the shSKA1 group compared with in the shCtrl group (Fig. 6B). In summary, these results suggest that silencing SKA1 expression in TE-1 cells may lead to cell cycle arrest at the G1/S phase and promote apoptosis.

SKA1-knockdown suppresses the migration ability of TE-1 cells. To determine the role of SKA1 expression in ESCC metastasis, the present study investigated the association between SKA1 expression and TE-1 cell migration ability. The results demonstrated that the number of migratory 
A
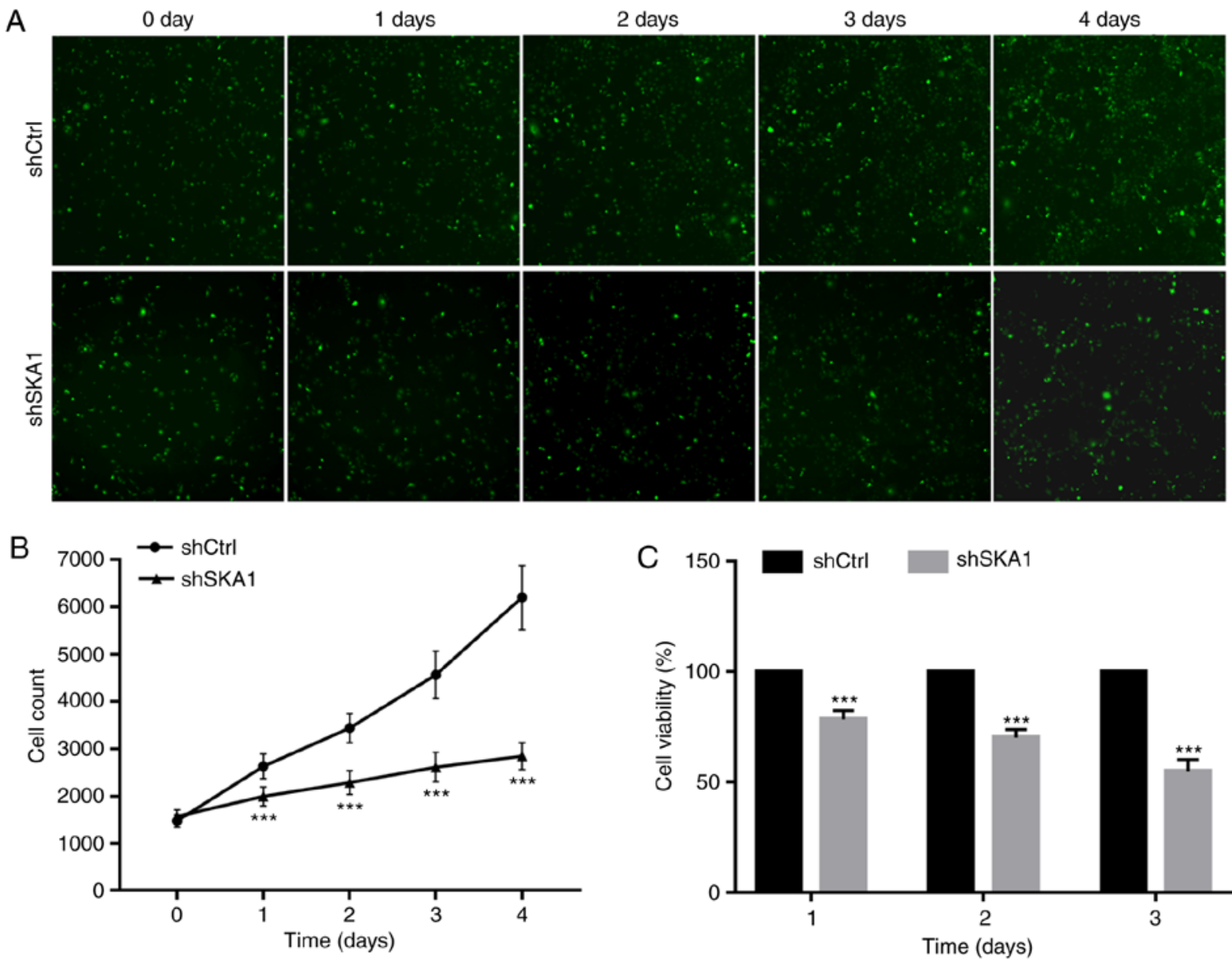

Figure 5. SKA1-knockdown significantly suppresses the proliferation of TE-1 cells. (A) Representative images (magnification, x100) and (B) cell counts determined using Celigo image cytometry. (C) The cell viability of TE-1 cells was assessed using a MTS assay. ${ }^{* * *}$ P $<0.001$ vs. shCtrl. SKA1, spindle and kinetochore-associated protein 1; shCtrl, cells transfected with control short hairpin RNA; shSKA1, cells transfected with SKA1 short hairpin RNA.

cells per field was significantly lower in the shSKA1 group compared with in the shCtrl group, indicating that the migration ability was significantly reduced in the cells lacking SKA1 expression compared with the control cells (Fig. 7).

\section{Discussion}

ESCC is a highly invasive type of malignant tumor with a high probability of lymph node metastasis. The incidence rate of ESCC is high worldwide, particularly in China (4). The identification of ESCC-associated oncogenes and the evaluation of their molecular and biological functions are critical to understand the molecular mechanism underlying the carcinogenesis of ESCC.

The present study identified that SKA1 expression was upregulated in $\sim 61.82 \%$ of ESCC tissues compared with CHEM tissues, as demonstrated by an IHC assay. In comparison with CHEM tissues, ESCC tissues demonstrated an increase in the mRNA expression of SKA1. SKA1 is localized on chromosome $18 \mathrm{q} 21.1$, is $\sim 30 \mathrm{kDa}$ in size and consists of 255 amino acid residues (17). SKA1 is an indispensable component essential for the stable binding of the kinetochore and microtubule. The formation of the SKA complex ensures the correct positioning of the spindle and kinetochore, and promotes transition throughout the process of mitosis (18-20). Evidence suggests that SKA1 may serve as a diagnostic molecular marker or independent prognostic factor for various malignant tumors. SKA1 expression is positively associated with recurrence, perineural invasion and poor survival in primary salivary adenoid cystic carcinoma (13). Furthermore, the expression of SKA1 has been reported to be increased in hepatocellular carcinoma and can serve as a prognostic marker (21). Consistent with the results of previous studies, the present study identified that SKA1 overexpression is associated with cancer cell differentiation, pathological $\mathrm{N}$ stage and pathological TNM stage in ESCC. These findings suggest that SKA1 overexpression may serve as an independent prognostic factor for ESCC.

SKA1 acts as an oncogene and is closely associated with the proliferation, apoptosis and migration of tumor cells. SKA1 regulates tumor development and knockdown of SKA1 expression results in inhibition of cell proliferation, induction of cell cycle arrest and promotion of apoptosis in hepatocellular carcinoma (22) and bladder cancer (23). Furthermore, knockdown of SKA1 expression also reduces the proliferation and migration of non-small cell lung cancer (24) and adenoid cystic carcinoma (25) cells. SKA1 promotes prostate tumorigenesis by enhancing centriole over-duplication and centrosome amplification (26). 

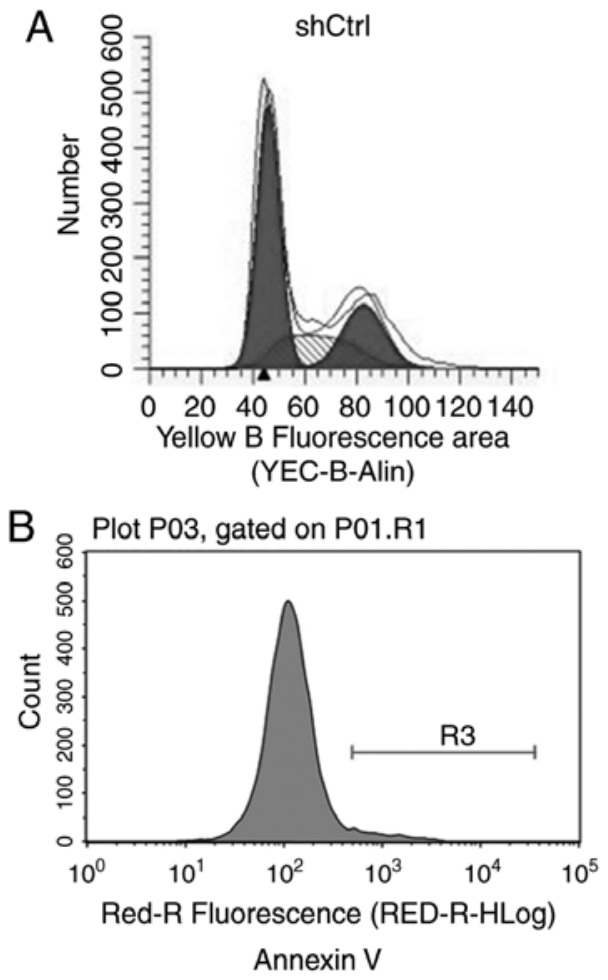
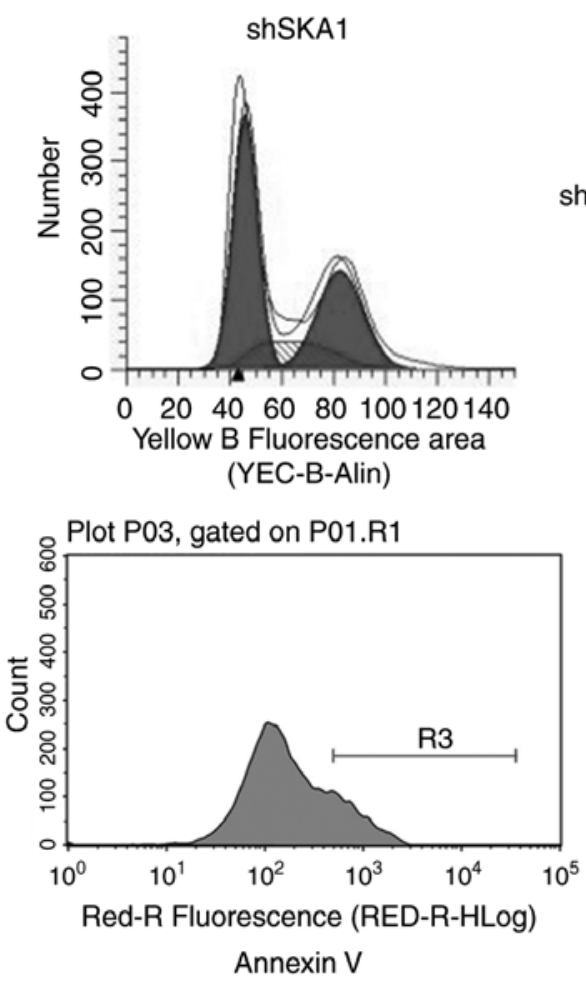
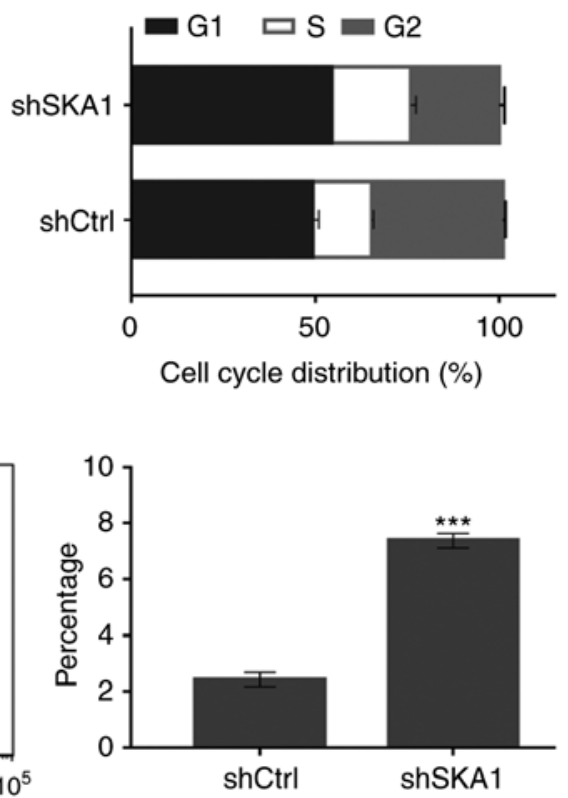

Figure 6. SKA1-knockdown arrests cycle progression and promotes TE-1 cell apoptosis. (A) Cell cycle progression was analyzed using flow cytometry. The bar graph represents the quantification of cells in the G1, S and G2 phases in each group. (B) Apoptosis was measured through flow cytometry analysis. The bar graph represents the quantification of apoptotic cells per group. ${ }^{* * * *} \mathrm{P}<0.001$ vs. shCtrl. SKA1, spindle and kinetochore-associated protein 1 ; shCtrl, cells transfected with control short hairpin RNA; shSKA1, cells transfected with SKA1 short hairpin RNA.
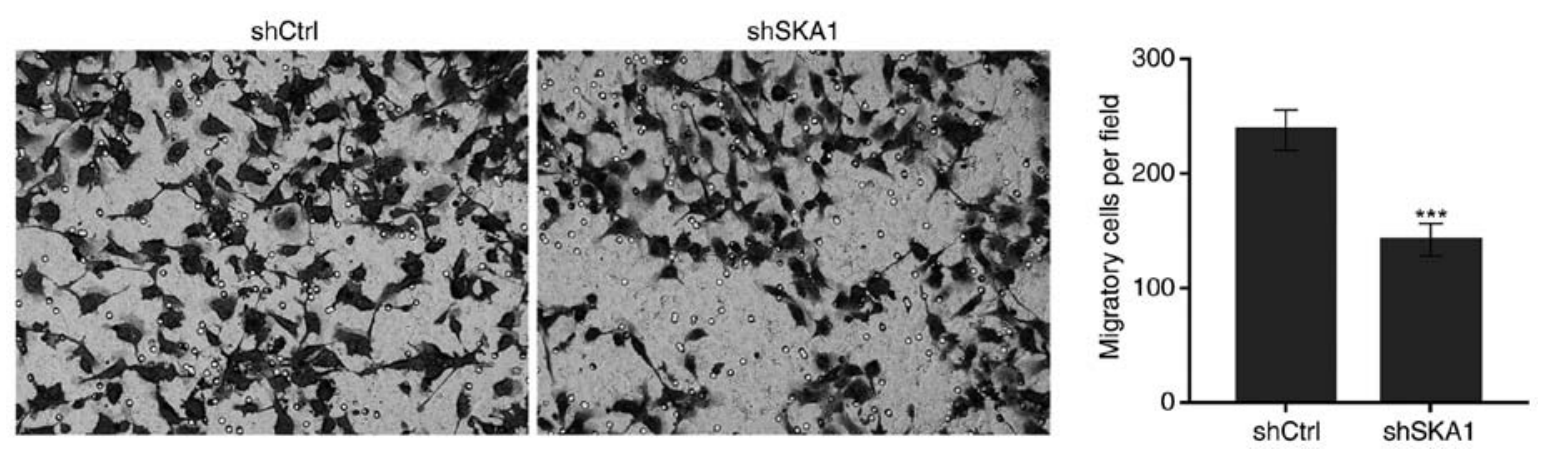

Figure 7. SKA1-knockdown significantly suppresses the migration of TE-1 cells. A cell migration assay was performed in a Transwell chamber without Matrigel. Representative images (magnification, x200) from triplicate experiments are presented. The bar graphs present the number of migratory cells for each group. ${ }^{* * *} \mathrm{P}<0.001$ vs. shCtrl. SKA1, spindle and kinetochore-associated protein 1; shCtrl, cells transfected with control short hairpin RNA; shSKA1, cells transfected with SKA1 short hairpin RNA.

However, to the best of our knowledge, no study has reported the function of SKA1 in ESCC. The present study identified that SKA1 expression is significantly increased in ESCC cells and that SKA1-silencing can inhibit cell proliferation and migration, arrest cell cycle progression, and promote cell apoptosis. These results suggest that SKA1 is an oncogene and that the downregulation of SKA1 expression may markedly inhibit tumorigenicity, which is consistent with the results of previous studies (22-25). In summary, these results suggest that SKA1 may be a potential therapeutic target for the targeted treatment of ESCC.

The present study had certain limitations. Firstly, in vivo experiments were not performed to further confirm the effect of SKA1-knockdown on the growth of subcutaneous tumors and lung metastasis. Secondly, the regulatory mechanism of SKA1 in ESCC was not studied. Future studies are required to further confirm the potential of SKA1 as a therapeutic target for the targeted treatment of ESCC.

In conclusion, the present study identified that SKA1 is overexpressed and serves as a prognostic marker in ESCC. SKA1-silencing suppressed the development of ESCC, suggesting that it could be a potential therapeutic target for the treatment of ESCC. However, further research is required to determine the mechanism underlying the upregulated expression of SKA1 in ESCC and its involvement in the generation and development of ESCC. Taken together, our findings indicate that SKA1 is a potential therapeutic target and a prognostic biomarker for ESCC. 


\section{Acknowledgements}

Not applicable.

\section{Funding}

The present study was supported by the Nature Science Foundation of Shandong Province (grant no. ZR2019PH034).

\section{Availability of data and materials}

Datasets analyzed during the current study are available from the corresponding author on reasonable request.

\section{Authors' contributions}

DH and MZ made substantial contributions to the conception and design of the study. DH, ZL and XL made substantial contributions to acquisition of data. DH and HF made substantial contributions to the analysis and interpretation of data. $\mathrm{DH}$ was involved in drafting the manuscript. MZ was involved in critically revising the manuscript for important intellectual content. All of authors have given final approval for the present version of the manuscript to be published.

\section{Ethics approval and consent to participate}

The Research Ethics Committee of Shandong Provincial Hospital Affiliated to Shandong University (Jinan, China) approved the study protocol, and all participants provided written informed consent.

\section{Patient consent for publication}

Not applicable.

\section{Competing interests}

The authors declare that they have no competing interests.

\section{References}

1. Bray F, Ferlay J, Soerjomataram I, Siegel RL, Torre LA and Jemal A: Global cancer statistics 2018: GLOBOCAN estimates of incidence and mortality worldwide for 36 cancers in 185 countries. CA Cancer J Clin 68: 394-424, 2018.

2. Chen W, Zheng R, Zeng H, Zhang S and He J: Annual report on status of cancer in China, 2011. Chin J Cancer Res 27: 2-12, 2015.

3. Chen W, Zheng R, Baade PD, Zhang S, Zeng H, Bray F, Jemal A Yu XQ and He J: Cancer statistics in China, 2015. CA Cancer J Clin 66: 115-132, 2016.

4. Arnold M, Soerjomataram I, Ferlay J and Forman D: Global incidence of oesophageal cancer by histological subtype in 2012 . Gut 64: 381-387, 2015.

5. Zeng H, Zheng R, Guo Y, Zhang S, Zou X, Wang N, Zhang L, Tang J, Chen J, Wei K, et al: Cancer survival in China, 2003-2005: A population-based study. Int J Cancer 136: 1921-1930, 2015.

6. Yang CS, Chen X and Tu S: Etiology and prevention of esophageal cancer. Gastrointest Tumors 3: 3-16, 2016.
7. Abnet CC, Arnold M and Wei WQ: Epidemiology of esophageal squamous cell carcinoma. Gastroenterology 154: 360-373, 2018.

8. Wang M, Smith JS and Wei WQ: Tissue protein biomarker candidates to predict progression of esophageal squamous cell carcinoma and precancerous lesions. Ann N Y Acad Sci 1434: 59-69, 2018.

9. Zulfiqar M, Bluth MH and Bhalla A: Molecular diagnostics in esophageal and gastric neoplasms: 2018 update. Clin Lab Med 38: 357-365, 2018.

10. Welburn JP, Grishchuk EL, Backer CB, Wilson-Kubalek EM, Yates JR III and Cheeseman IM: The human kinetochore Ska1 complex facilitates microtubule depolymerization-coupled motility. Dev Cell 16: 374-385, 2009.

11. Schmidt JC, Arthanari H, Boeszoermenyi A, Dashkevich NM, Wilson-Kubalek EM, Monnier N, Markus M, Oberer M, Milligan RA, Bathe M, et al: The kinetochore-bound Ska1 complex tracks depolymerizing microtubules and binds to curved protofilaments. Dev Cell 23: 968-980, 2012.

12. Dong C, Wang XL and Ma BL: Expression of spindle and kinetochore-associated protein 1 is associated with poor prognosis in papillary thyroid carcinoma. Dis Markers 2015: 616541, 2015.

13. Zhao L, Jiang L, Du P, Zhang D, Liu Z, Li K and Zhang B: Expression of SKA1 and MMP-9 in primary salivary adenoid cystic carcinoma: Correlation with tumor progression and patient prognosis. Acta Otolaryngol 136: 575-579, 2016.

14. Wu J, Chai Y, Zhou XM, Chen QX and Yan FL: Ivor Lewis esophagectomy with two-field lymph node dissection for squamous cell carcinoma of the lower thoracic esophagus. Ai Zheng 26: 307-311, 2007.

15. Shi M, Wang Z, Liu XY and Chen D: Inactivation of RUNX3 predicts poor prognosis in esophageal squamous cell carcinoma after Ivor-Lewis esophagectomy. Med Oncol 31: 309, 2014.

16. Livak KJ and Schmittgen TD: Analysis of relative gene expression data using real-time quantitative PCR and the 2(-Delta Delta C(T)) method. Methods 25: 402-408, 2001.

17. Sauer G, Körner R, Hanisch A, Ries A, Nigg EA and Silljé HH: Proteome analysis of the human mitotic spindle. Mol Cell Proteomics 4: 35-43, 2005.

18. Park JE, Song H, Kwon HJ and Jang CY: Ska1 cooperates with DDA3 for spindle dynamics and spindle attachment to kinetochore. Biochem Biophys Res Commun 470: 586-592, 2016.

19. Thomas GE, Bandopadhyay K, Sutradhar S, Renjith MR, Singh P, Gireesh KK, Simon S, Badarudeen B, Gupta H, Banerjee M, et al: EB1 regulates attachment of Ska1 with microtubules by forming extended structures on the microtubule lattice. Nat Commun 7 : 11665, 2016.

20. Abad MA, Zou J, Medina-Pritchard B, Nigg EA, Rappsilber J, Santamaria A and Jeyaprakash AA: Ska3 ensures timely mitotic progression by interacting directly with microtubules and Ska1 microtubule binding domain. Sci Rep 6: 34042, 2016.

21. Chen Y, Zhao J, Jiao Z, Wang W, Wang D, Yu X, Shi Z, Ge N, Pan Q, $\mathrm{Xia} \mathrm{J}$, et al: SKA1 overexpression is associated with poor prognosis in hepatocellular carcinoma. BMC Cancer 18: 1240, 2018.

22. Qin X, Yuan B, Xu X, Huang H and Liu Y: Effects of short interfering RNA-mediated gene silencing of SKA1 on proliferation of hepatocellular carcinoma cells. Scand J Gastroenterol 48: 1324-1332, 2013

23. Tian F, Xing X, Xu F, Cheng W, Zhang Z, Gao J, Ge J and Xie H: Downregulation of SKA1 gene expression inhibits cell growth in human bladder cancer. Cancer Biother Radiopharm 30: 271-277, 2015.

24. Shen L, Yang M, Lin Q, Zhang Z, Miao C and Zhu B: SKA1 regulates the metastasis and cisplatin resistance of non-small cell lung cancer. Oncol Rep 35: 2561-2568, 2016.

25. Zhao LJ, Yang HL, Li KY, Gao YH, Dong K, Liu ZH, Wang LX and Zhang B: Knockdown of SKA1 gene inhibits cell proliferation and metastasis in human adenoid cystic carcinoma. Biomed Pharmacother 90: 8-14, 2017.

26. Li J, Xuan JW, Khatamianfar V, Valiyeva F, Moussa M, Sadek A, Yang BB, Dong BJ, Huang YR and Gao WQ: SKA1 over-expression promotes centriole over-duplication, centrosome amplification and prostate tumourigenesis. J Pathol 234: 178-189, 2014. 